\title{
Dosimetric comparison of different radiation techniques (IMRT vs. 3-dimensional) of the "true" (deep) ano-inguinal lymphatic drainage of anal cancer patients
}

Hendrik Dapper ${ }^{1 *}$, Markus Oechsner ${ }^{1}$, Christoph Hirche ${ }^{2}$, Stefan Münch ${ }^{1}$, Christina Sauter ${ }^{1}$, Kai Borm¹, Jan C. Peeken', Stephanie E. Combs ${ }^{1,3,4}$ and Daniel Habermehl ${ }^{1,3}$

\begin{abstract}
Introduction: The ano-inguinal lymphatic drainage (AILD) is located in the subcutaneous adipose tissue of the proximal medial thigh. Currently, there are no recommendations for an inclusion of the 'true' AlLD in the clinical target volume (CTV) of definitive chemoradiation for anal cancer patients. To estimate the relevance of inguinal recurrence, we compared the incidental dose to the AlLD in anal cancer (AC) patients who were treated either with Volumetric Arc Therapy - Intensity Modulated Radiation Therapy (VMAT-IMRT) or conventional 3D-radiation technique.

Methods: One VMAT-IMRT-plans and one 3D-plans were calculated on the same target volumes and identical dose prescription in ten patients. We defined the volume of the AlLD on the planning CT-scans based on the information of new fluorescence methods. Furthermore, we defined several anatomical subvolumes of interest inside the AlLD. We examined and compared absolute and relative dosimetric parameters of the AILD and different anatomical subunits.

Results: The Dmean of the AILD was 40 Gy in the 3D-group and 38 Gy in the IMRT-group. Dmean and Dmedian as well as the V30Gy of the AILD and all subvolumes of the caudal AILD were significant higher using 3D-RT compared to IMRT. Even though the absolute differences were small, in the caudal aspect of the ano-inguinal lymphatic drainage the V30Gy could be more than $10 \%$ less with VMAT-IMRT.

Conclusions: 3D-RT was slightly superior to IMRT in terms of dose coverage of the AlLD. However, the absolute differences were very small. Some relevant caudal parts of the AILD received an insufficient dose for treating potential micrometastases. Particularly in high-risk situations, this may lead to inguinal recurrence and therefore the true deep AlLD should be included into the target volume in high risk patients.
\end{abstract}

Keywords: Anal Cancer, Ano-inguinal lymphatic drainage, Dosimetric quantification, IMRT

\section{Purpose}

A combined chemoradiation (CRT) protocol using IMRT is the standard treatment regimen for anal cancer $[1,2]$. The anatomical definition of the lymphatic drainage of the anus to the inguinal region is complex, and the literature on this is inconsistent. In particular, the ano-inguinal lymphatic drainage (AILD) pathway has long been undetectable with

\footnotetext{
* Correspondence: hendrik.dapper@mri.tum.de

The work was presented at ESTRO 2018.

'Department of Radiation Oncology, Klinikum rechts der Isar, TU München, Ismaninger Str. 22, 81675 Munich, Germany

Full list of author information is available at the end of the article
}

traditional lymphangiography due to very small lymphatic canals [3]. Therefore, the AILD has not been properly taken into consideration for a long time, and inclusion of the elective CTV of the AILD is not recommended by standard contouring guidelines. Furthermore, CTV-recommendations are generally based not on the "anatomical" drainage but on the incidence of nodal metastases in a particular site $[4,5]$. In the last years, new fluorescence-imaging methods have been developed. Those help to define the area of the AILD in real-time and transcutaneously [6, 7]. We recently published an analysis in which, for the first time, surgeons and radiation oncology specialists, presented a clearly defined

(c) The Author(s). 2018 Open Access This article is distributed under the terms of the Creative Commons Attribution 4.0 International License (http://creativecommons.org/licenses/by/4.0/), which permits unrestricted use, distribution, and 
anatomically area for the "true" AILD based on fluorescence-imaging method. We evaluated the dose distribution of VMAT-IMRT-treated anal cancer patients to this defined volume, showing that, in particular, the caudal areas of inguinal lymphatic drainage did not receive an adequate prophylactic dose for micrometastatic spread [8].

The clinical relevance of inguinal lymph nodes in anal carcinoma is not to be overlooked. Especially among patients with higher T-staging (T3 - T4) and those with uninvolved inguinal nodes at the time of diagnosis, who did not receive elective radiation to the groin, the inguinal recurrence rates can exceed $30 \%$ [9-13]. If elective $3 \mathrm{D}$-radiation therapy to the groin was performed without inclusion of the AILD, inguinal lymph node relapse would be considered quite rare. In an analysis of 167 patients treated between 1996 and 2004, Das et al. reported only one inguinal relapse $(0.6 \%)$ in a patient with history of ipsilateral inguinal nodal involvement [14]. The risk of inguinal lymph node recurrence in IMRT treated patients is difficult to assess, as there are just a few studies demonstrating patterns of recurrence. However, even if the inguinal relapse rate in the study with most patients (106) was low (about 4\%), the results indicate that using IMRT could lead to increased numbers of recurrence, especially if inguinal lymph node metastases were present at diagnosis (3 of 4 cases with relapse) [15]. Compared to 3D-techniques and field arrangements, due to steep dose gradients, IMRT offers the opportunity of dose-sparing to organs at risk (OAR) at similar loco-regional control rates [16, 17]. Even though IMRT is the recommended standard by now, using this new technique we have already experienced marginal misses in head and neck and also ano/rectal cancer patients due to misunderstandings of anatomical conditions (e. a. perirectal, presacral) [18-20].

Inguinal recurrence in IMRT-treated patients occurs mostly in patients who already had positive inguinal nodes at diagnosis. This fact raises the question, whether recurrence is due to insufficient dose to inguinal macroscopic involved nodes or an insufficient dose to potential micrometastases in AILD. To prove the impact of radiation techniques, we compared IMRT- versus 3D-techniques in anal cancer patients regarding dose distribution to the AILD. Our aim was to estimate whether the change to IMRT as the new standard technique for anal carcinoma patients could have led to insufficient dose for the treatment of micrometastases in the area of the AILD.

\section{Methods}

\section{Patient selection and radiation technique details}

We selected ten patients with a diagnosis of anal cancer and clinical not involved inguinal nodes who were already treated with primary chemoradiation protocol between 2012 and 2017. We prospectively generated a standardized elective clinical target volume for anal cancer as recommended by RTOG [5] on the original planning CT scan with $3 \mathrm{~mm}$ slice thickness for each patient. All patients were in prone position. Subsequently, we calculated two plans for each individual, one for volumetric arc therapy (VMAT) and one 3-dimensional (3D)-plan. All plans were created for a Varian Clinac ${ }^{\circ}$ DHX linear accelerator (Varian Medical Systems, Palo Alto, CA, USA). Dose prescription for both radiation techniques was $36 \mathrm{~Gy}$ (1.8 Gy single dose) to PTV1, which included the primary tumor region (PTR), the elective pelvic lymph nodes and the inguinal nodes and subsequently $14.4 \mathrm{~Gy}$ (1.8 Gy single dose) to PTV2, which includes the PTR and the elective pelvic nodes without inguinal lymph nodes. Aim was, to cover the primary tumor site and the pelvic lymph nodes with a total dose of $50.4 \mathrm{~Gy}$ and the inguinal lymph nodes with a total dose of $36 \mathrm{~Gy}$ (single dose $1.8 \mathrm{~Gy}$ ). Dose constraints for organs at risk (OAR) (rectum, sigmoid, small bowel, femoral head left \& right, penis/scrotum or vagina/vulva, skin of AILD, urinary bladder) orientated on Quantitative Analyses of Normal Tissue Effects in the Clinic (QUANTEC) [21].

For VMAT, regularly 3 arcs in the main plan (PTV1) and 2 arcs for the boost plan (PTV2) (6 or $15 \mathrm{MV}$ ) were used. The dose was prescribed to the median of the PTV (ICRU83).

For 3D-radiation-plans, we utilized 6-12 fields with wedges $\left(15-45^{\circ}\right)$. Main fields were planned from posterior and lateral. Segment fields were used to improve dose coverage and dose homogeneity. The 3D planning was done according to the technique used in the past. Analogous to VMAT, the dose was prescribed to the median of the PTV (ICRU83). For both techniques we used Eclipse 13.0 Treatment Planning System (Varian Medical Systems, Palo Alto, CA, USA) for contouring and dose comparison.

\section{Definition of the AILD}

We defined the AILD on each patient almost identical to our previous AILD-fluorescence-study (Fig. 1a) [8]. The cranial border of the AILD (the upper ischioanal fossa) was the origin of the levator ani muscle. The caudal demarcation was defined $3 \mathrm{~cm}$ below the lower end of the anal canal. On inguinal site, the ventral and medial demarcation was the skin of the medial thigh; dorsal was the connection line of the dorsal edge of the gluteal muscles. The lateral demarcations were the adductor muscles (anal) or the medial edge of the sartorius or iliopsoas adductor muscles (inguinal).

In our previous study we demonstrated, that especially the caudal parts of the AILD were insufficiently covered by the $30 \mathrm{~Gy}$ isodose in IMRT-treated patients [8]. In contrast, the cranial parts of the AILD (ischioanal fossa) 


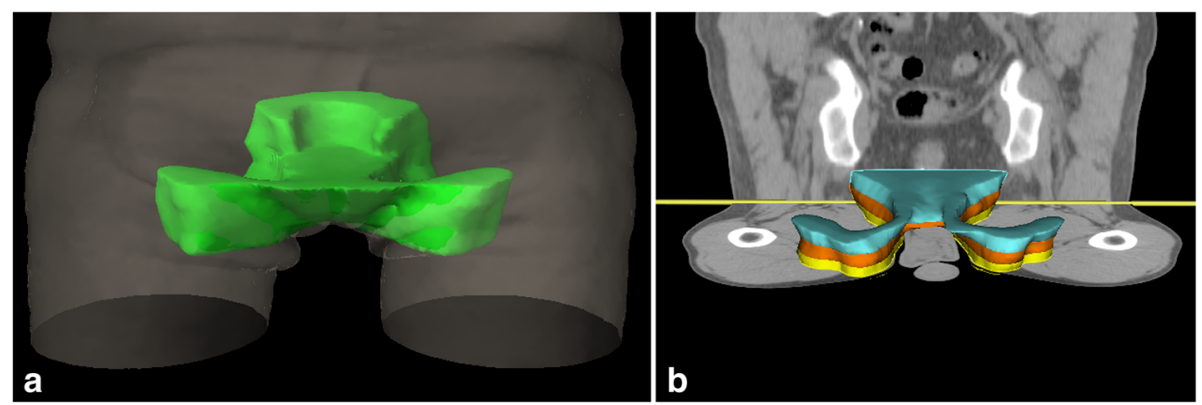

Fig. 1 The ano-inguinal lymphatic drainage (a) consist of a dorsal/cranial (ischiorectal fossa) and a ventral/caudal part (subcutaneous tissue from the anus to inguinal site). The caudal part is currently not included into the elective CTV of the recommendations by RTOG. For dose comparison we divided the caudal part in three different Levels $(\mathbf{b})$, each with a longitudinal extension of $1 \mathrm{~cm}$

were adjacent to the anal canal and usually covered by higher doses. The interesting part of the AILD begins where the first subcutaneous connective tissue connection from the area of the anus to the inguinal lymph nodes below the pubic bone exists. This connection is not included into the CTV in various established contouring guidelines $[5,22,23]$. Therefore, we also compared the dose of different subvolumes of the AILD: We divided the AILD into a cranial part (AILDcranial $=$ ischiorectal fossa) and a caudal part (AILDcaudal $=$ first $3 \mathrm{~cm}$ below the anal verge). Furthermore, we divided the AILDcaudal into three different levels, each of $1 \mathrm{~cm}$ longitudinal extension (Fig. 1b).

\section{Dosimetric evaluation}

We compared absolute (Dmean, Dmedian, D98\%, D2\%) and relative dose parameters (V10-V50) of the AILD for both radiation techniques. In addition, we analyzed different dose parameters to the AILDcranial, AILDcaudal and Level1, Level2 and Level3, as represented in the dose-volume histogram.

For all dose parameters of the OAR, a two-sided Wilcoxon test was performed with SPSS 25.0 (SPSS Inc., Chicago, IL, USA) to identify significant differences between the plans for several dose parameters. A $p$-value $<0.05$ was considered to indicate statistical significance.

\section{Results}

\section{Patient characteristics}

Nine of the ten patients had anal canal cancer, whereas one patient had a T2 tumor of the anal margin. There were two patients with $\mathrm{T} 1$, four patients with $\mathrm{T} 2$ and two patients with T3 disease. Lymph node involvement could be found in four patients. All positive nodes were located in pelvic lymph nodes above the primary tumor. In both groups the PTV was well covered by the prescription dose of $36 \mathrm{~Gy}$ (PTV1) and 14.4Gy (PTV2). There was no statistically significant difference between these parameters $(p=0.912)$. The measured volume of the AILD was $943 \mathrm{cc}$. Dose constraints for organs at risk (OAR) orientated on Quantitative Analyses of Normal Tissue Effects in the Clinic (QUANTEC) were respected for both radiation techniques.

\section{Dosimetric analysis of the whole volume of the AILD}

The dose parameters of the AILD hardly differed between the two irradiation techniques (Table 1). Both, the Dmean and the Dmedian of the AILD were significant lower using IMRT compared to 3D-RT ( $p=0.008 ; 9=0.017)$, even if the absolute differences were just $1.8 \mathrm{~Gy}$ for Dmean and 3.3 Gy for Dmedian. Ninety-eight percent of this volume was covered by $34 \mathrm{~Gy}$ (IMRT) and $37 \mathrm{~Gy}(3 \mathrm{D})(p=0,075)$. There were no relevant differences in D98\% however, there was a small but significant difference in the D2\% (VMAT: 51.8 Gy, 3D-RT: 53.2).

Only in low dose range, the IMRT group had a non-significant higher coverage of the AILD (96\%) compared to 3D-radiation (87\%). With higher doses (V20Gy-V50Gy), more volume of the AILD was covered in the 3D-group, though the differences never exceeded $6 \%$. The V10-V40Gy were significantly higher with $3 \mathrm{D}$ conformal technique $(p=0.008-0.021)$. Eighty-nine percent of the AILD was covered with $20 \mathrm{~Gy}, 79 \%$ with 30 Gy and $61 \%$ with 40 Gy using 3D-RT, whereas $86 \%$ of the AILD was covered with $20 \mathrm{~Gy}, 73 \%$ with 30 Gy and $54 \%$ with 40 Gy using IMRT.

\section{Dosimetric analysis of different subvolumes of the AILD}

With a subdivision into different regions of interest, the AILD can be examined more precisely (Table 2). The cranial aspect of the AILD (AILDcranial), which largely corresponds to the ischio-rectal fossa, encloses the anal canal and thus the PTV. Unsurprisingly, here the Dmean and Dmedian almost reached the prescription dose of $50.4 \mathrm{~Gy}$ regardless of the radiotherapy technique, and 98 $\%$ of the cranial volume was covered by 34 Gy (IMRT) and $37 \mathrm{~Gy}(3 \mathrm{D})(p=0,075)$. Also the clinical relevant V30Gy reached almost $100 \%$ of the volume. 
Table 1 Dose statistics of the ano-inguinal lymphatic drainage (AILD) with two different radiation techniques

\begin{tabular}{llllll}
\hline Technique & \multicolumn{5}{c}{} \\
\hline & Dose (Gy) & & & & \\
& Dmean & Dmeadian & D98\% & D2\% & \\
3D-RT & 40,2 & 44,8 & 12,5 & 53,2 & \\
IMRT & 38,0 & 41,5 & 11,6 & 51,8 & \\
p-value & 0,008 & 0,017 & 0,441 & $\mathbf{0 , 0 0 7}$ & \\
& Volume (\%) & & & & \\
& V10Gy & V20Gy & V30GY & V40Gy & V50Gy \\
3D-RT & 87,1 & 88,5 & 78,8 & 61,0 & 31,3 \\
IMRT & 95,6 & 85,3 & 73,1 & 54,2 & 26,9 \\
p-value & 0,735 & $\mathbf{0 , 0 2 1}$ & $\mathbf{0 , 0 1 1}$ & $\mathbf{0 , 0 0 8}$ & $\mathbf{0 , 0 6 6}$ \\
\hline
\end{tabular}

$R T$ radiation therapy, Gy gray, 3D 3 dimensional, IMRT intensity modulated radiation therapy

Boldface indicates values that are statistically significant

The interesting region is the caudal part of the AILD (AILDcaudal), which cranially starts at the level of the anal verge and falls below $3 \mathrm{~cm}$ of the anus and inguinal nodes. Larger shares of this region are below or between the target volumes proposed by the RTOG. This is also reflected in the dose parameters. The Dmean and Dmedian were 32-35 Gy, independently of the radiation techniques, with slight but significantly higher values using 3D-radiotherapy. Ninety-eight percent of the volume was just covered by 11 Gy (both groups). Only a bit more than half of volume in the IMRT-group and two third of the volume in the 3D-group were covered by 30Gy $(p=0.011)$. The most cranial level (Level1) of AILDcaudal was partly overlapping with the PTV of the primary tumor and of the elective inguinal target volume in all patients. Here, the Dmean and Dmedian in both groups were at least 40 Gy and did not differ much from each other, although the slightly higher values in the $3 \mathrm{D}$-group were statistically significant. Also Level2, which represents the volume which is $1-2 \mathrm{~cm}$ caudally of the anal verge, was partly covered with the PTV, which is shown in the D2\% > 51 Gy in both groups. The Dmean and Dmedian, which were both significantly higher with 3D-RT, exceeded 33 Gy with IMRT and 36Gy with 3D-RT. Compared to Level1, the V30Gy was $18 \%$ less using IMRT (63\%) and $12 \%$ less using 3D-RT (73\%). The huge dose drop appeared starting $2 \mathrm{~cm}$ below the anal verge (Level3). The minimal dose (D2\%) fell below 50 Gy. The Dmean and Dmedian were smaller 30Gy for either technique. Only one third of Level3 received 30 Gy utilizing 3D-RT, whereas the V30Gy was just $25 \%$ with IMRT.

\section{Discussion}

In the current study we were able to show that the Dmean and Dmedian as well as the V30Gy of the AILD and all subvolumes of the caudal AILD were significantly higher using 3D-RT compared to IMRT. Even though the absolute differences were small in the caudal aspect of the ano-inguinal lymphatic drainage, the V30Gy could be more than $10 \%$ less with VMAT-IMRT. With both

Table 2 Dose statistics of different subvolumes inside the ano-inguinal lymphatic drainage (AILD) with two different radiation techniques

\begin{tabular}{|c|c|c|c|c|c|c|}
\hline \multirow[t]{2}{*}{ Structure } & \multirow[t]{2}{*}{ Technique } & \multicolumn{4}{|c|}{$\underline{\text { Dose (Gy) }}$} & \multirow{2}{*}{$\begin{array}{l}\text { Volume (\%) } \\
\text { V30Gy }\end{array}$} \\
\hline & & Dmean & Dmeadian & D98\% & D2\% & \\
\hline \multirow[t]{3}{*}{ AlLDcranial } & 3D-RT & 49,0 & 50,7 & 36,9 & 53,3 & 98,7 \\
\hline & IMRT & 47,3 & 49,5 & 33,6 & 51,9 & 98,5 \\
\hline & $p$-value & 0,008 & 0,008 & 0,075 & 0,008 & 0,236 \\
\hline \multirow[t]{3}{*}{ AlLDcaudal } & 3D-RT & 34,7 & 35,4 & 11,3 & 52,3 & 66,5 \\
\hline & IMRT & 32,1 & 32,0 & 10,7 & 51,4 & 57,0 \\
\hline & $p$-value & 0,011 & 0,011 & 0,514 & 0,018 & 0,011 \\
\hline \multirow[t]{3}{*}{ Level1 } & 3D-RT & 41,2 & 42,9 & 22,4 & 52,5 & 85,3 \\
\hline & IMRT & 39,5 & 40,4 & 21,6 & 51,6 & 81,0 \\
\hline & $p$-value & 0,013 & 0,015 & 0,285 & 0,011 & 0,110 \\
\hline \multirow[t]{3}{*}{ Level2 } & 3D-RT & 36,5 & 37,4 & 15,4 & 51,9 & 73,4 \\
\hline & IMRT & 33,8 & 33,7 & 15,6 & 51,4 & 62,6 \\
\hline & $p$-value & 0,011 & 0,012 & 0,722 & 0,183 & 0,008 \\
\hline \multirow[t]{3}{*}{ Level3 } & 3D-RT & 26,3 & 25,0 & 9,9 & 48,5 & 36,0 \\
\hline & IMRT & 22,3 & 20,1 & 9,5 & 44,4 & 25,2 \\
\hline & $p$-value & 0,011 & 0,015 & 0,813 & 0,173 & 0,011 \\
\hline
\end{tabular}

$R T$ radiation therapy, AILD ano-inguinal lymphatic drainage, Gy gray, $3 D$ dimensional, IMRT intensity modulated radiation therapy Level1: $1 \mathrm{~cm}$ of AILD caudally of the anal verge, Level2: $1-2 \mathrm{~cm}$ of AILD caudally of the anal verge, Level3: $3 \mathrm{~cm}$ of AILD caudally of the anal verge Boldface indicates values that are statistically significant 
techniques a relevant volume of the AILD was not covered by $30 \mathrm{~Gy}$. The results for the whole volume of the AILD were similar to our study in which we retrospectively analyzed the dose to the AILD in IMRT-treated patients [8]. Although the dose prescription in this study was slightly different, the Dmean was $41 \mathrm{~Gy}$ and V30Gy was $76 \%$ compared to 38 Gy and senventy- $3 \%$ in the present study.

\section{Interpretation of dose distribution in context with anatomical conditions of the AILD}

We chose the V30Gy as an important dose parameter because RTOG98-11 used 30.6 Gy for elective irradiation of the groin in patients with uninvolved inguinal nodes and therefore should be considered as sufficient for treatment of micrometastases. The V30Gy of the AILD was significant higher for 3D-RT (79\%) than for IMRT-treated patients (73\%). However, in some critical areas of the caudal AILD (Level2), the differences reached more than $10 \%$ (73.4\% versus $62.6 \%$ ).

The direct drainage from the anus to inguinal site is on ventral site of the perineal pouch. Below the pubic symphysis, branches of the pudendal vessels and the first fat tissue components of the ischioanal fossa reach the external genitalia following the adductor muscles (lateral) and the skin (medial) [24]. We chose this area as the first level of our anatomically detailed breakdown of dose distribution (Level1). The anus and inguinal site at this level were covered by the previous PTV and received adequate dose with both radiation techniques. For this critical level, the V30Gy was $85 \%$ with 3D-RT and only $4 \%$ lower (81\%) with IMRT. The absolute differences are indeed small and it would be daring to assume that these small differences increase the risk of inguinal recurrences.

Around $1 \mathrm{~cm}$ below this level we defined Level2. The differences in dose distribution between the two techniques increased. Figure 2 shows the $30 \mathrm{~Gy}$ isodose (color wash) in a selected patient using 3D-RT (2a) or IMRT (2b). The caudal parts of the AILD were properly covered with 3D-RT, whereas in IMRT the V30Gy was more conformal surrounding the PTV, and less volume of the AILD was covered. Ultimately, this region could be the cause of regional recurrences in some patients with high risk tumors treated with IMRT. With immune-fluorescence methods we were previously able to show the AILD to fall about $3 \mathrm{~cm}$ below the level of the anal verge. This level (Level3) is the most caudal potential lymphatic drainage of the anal verge and unlikely to contain micrometastases in low risk tumors of the anal canal. Since inguinal recurrences rarely occur $3 \mathrm{~cm}$ below the primary tumor, we do not expect this level to be responsible for inguinal relapse in the vast majority of cases. Nevertheless, in high risk anal cancer, especially for big tumors $(\geq \mathrm{T} 3)$ of the anal verge with a high number of positive inguinal and pelvic lymph nodes at diagnose, this part of the AILD could be of clinical relevance. The change from 3D-RT to IMRT might have an impact regarding inguinal recurrence in those patients.

\section{Clinical relevance of elective radiation of lymphatic-drainage} The most common grade 3 or 4 toxicity in the two big prospective trials (2D- or $3 \mathrm{D}$-techniques) was radio dermatitis. In both trials (RTOG98-11 and ART II) grade $\geq 3$ skin toxicity was $48 \%$ in the mitomycin-based arm $[1,2]$. Scher et al. summarized 8 IMRT-studies which demonstrated skin toxicity after radiation of anal cancer patients $(n=39-78)$ [25]. Dose to the inguinal lymph nodes was mainly $45 \mathrm{~Gy}$. Grade $\geq 3$ skin toxicity ranged from zero to $42 \%$ (mean: 23\%). With IMRT-techniques $\geq 3$, skin toxicity was significantly reduced in RTOG-0529 $(23 \%)$, whereas in other IMRT-studies it could reach $69 \%$ $[15,26,27]$. Because of the fact that the critical part of the ano-inguinal lymphatic drainage is located subcutaneously on the medial thigh, we would expect an increase in this relevant toxicity. Furthermore, the genito-urinary side effects might increase [28].

In order to ascertain the clinical benefit of inclusion of the AILD into the CTV, the number and pattern of

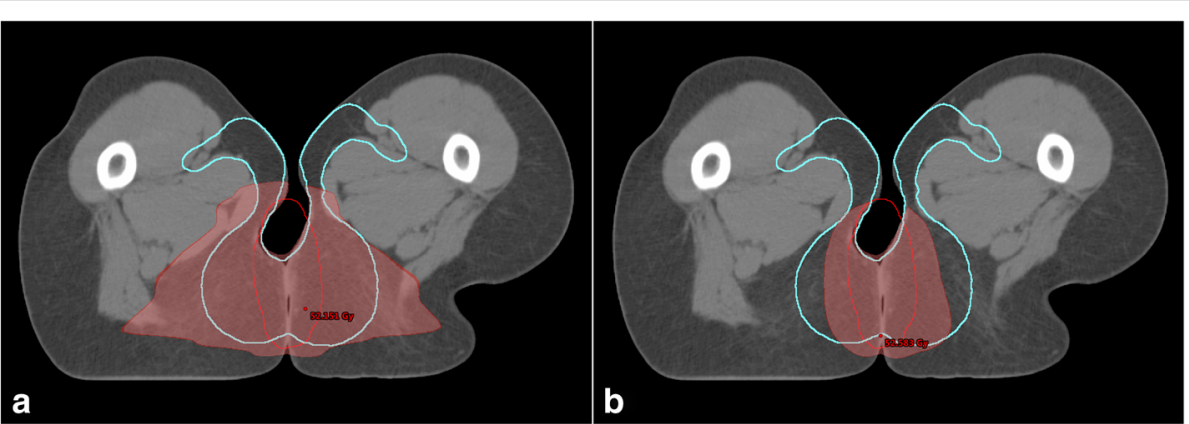

Fig. 2 Axial CT slide $1.5 \mathrm{~cm}$ below the lower end of the anal canal in one patient. $30 \mathrm{~Gy}$ is represented in colorwash (red area). In this case, about $75 \%$ of ano-inguinal lymphatic drainage (cyan) is covered with 3D-RT (a) and just about 50\% is covered using IMRT (b) 
recurrence are important. Overall, the data on IMRTtreated patients and explicit inguinal recurrences and patterns of spread are less well explored. In the RTOG0529-study, loco regional failure was $13 \%$ after 4 years. The Study did not show any detailed differentiation of the site of failure, though [17]. Only Tomasoa et al. provided detailed data regarding the number and patterns of inguinal recurrence. In a retrospective analysis of 106 patients treated by SIB-IMRT, four patients had inguinal relapse (4\%) [15]. Three out of these four patients had initially involved inguinal nodes. A total dose to lymph nodes of 49.5 à $1.5 \mathrm{~Gy}$ was given, which means a higher biological dose than in conventional techniques. The authors (Tomasoa et al.) compared this to similar results of Wright et al. (3-field technique) and mentioned that more conformal techniques (IMRT) with higher total dose might not reduce inguinal relapse $[15,29]$. This indicates that microscopic disease in the AILD but not the lymph nodes themselves might be responsible for inguinal relapse. Another important finding of these studies was that none of inguinal recurrences occurred below approximately $1 \mathrm{~cm}$ of the level of the anal canal. Wright et al. however, could show that inguinal recurrences arise up to $3 \mathrm{~cm}$ below the anal verge (conventional technique) [29]. Up to this date, insufficient data concerning inguinal recurrence in anal carcinoma is available. However, there are a few cases of inguinal relapse even in inguinal-treated patients. To balance the relevant skin toxicity with the risk of inguinal relapse in high risk tumors (T3-T4, inguinal involvement at diagnosis, anal verge), an inclusion of the AILD into the target volume could be useful.

\section{Conclusion}

3D-RT was slightly superior to IMRT in terms of dose coverage of the AILD. However, the absolute differences were very small. Some relevant caudal parts of the AILD received an insufficient dose for treating potential micrometastases. Particularly in high-risk situations, this may lead to inguinal recurrence and therefore the true deep AILD should be included into the target volume in high risk patients.

\section{Abbreviations \\ 2D/3D: 2/3 dimensional; AlLD: Ano-inguinale lymphatic drainage; CRT: Chemoradiation therapy; CTV: Clinical target volume; E. a.: For example; Gy: Gray; IMRT: Intensity modulated radiotherapy; LD: Lymphatic drainage; OAR: Organs at risk; PET: Positron emission tomography; PTR: Primary tumor region; PTV: Planning target volume; RT: Radiation therapy; SIB: Simultaneously integrated boost; VMAT: Volumetric modulated arc therapy}

\section{Acknowledgements}

Not applicable

\section{Funding}

This research did not receive any specific grant from funding agencies, the public, commercial, or not-for-profit sectors.

\section{Availability of data and materials}

The present data is summarized in this paper (METHODS). The complete dataset can be retrieved from the authors upon formal request from interested readers.

\section{Authors' contributions}

$\mathrm{DH}$ and SEC treated the patients and provided the data and study infrastructure. DH and HD developed the study design, collected, and interpreted data, performed statistical analysis and drafted the manuscript. DH made substantial contributions to conception and design of the study, interpreted data and revised the manuscript. MO calculated the different radiation plans (IMRT and 3D-RT). CH, CS, SM, KB, JCP and SEC contributed significantly to the discussion and interpretation of the results. $\mathrm{CH}$ made the basic research regarding fluorescence detection of the AlLD. HD and $\mathrm{DH}$ made the main contributions to conception and design of the study, analysed and interpreted data and drafted the manuscript. All co-authors read and revised the manuscript. The final version of the manuscript was approved by all co-authors.

\section{Ethics approval and consent to participate}

The study was performed in accordance with the ethics standards at the Techniqueal University of Munich (TUM) (ethical vote: 28.02.2018s, 88/18 S). Name of committee: Ethikkommission der Technischen Universität München.

Consent for publication

Not applicable

\section{Competing interests}

The authors declare that they have no competing interests.

\section{Publisher's Note}

Springer Nature remains neutral with regard to jurisdictional claims in published maps and institutional affiliations.

\section{Author details}

'Department of Radiation Oncology, Klinikum rechts der Isar, TU München, Ismaninger Str. 22, 81675 Munich, Germany. ${ }^{2}$ Department for Hand-, Plastic and Reconstructive Surgery, Burn Centre, BG-Trauma Centre Ludwigshafen/ Rhine, University of Heidelberg, Ludwig-Guttmann-Str. 13, 67071 Ludwigshafen, Germany. ${ }^{3}$ Institute for innovative Radiotherapie (iRT), Helmholtz Zentrum München, Ingolstädter Landstr. 1, Neuherberg, Germany. ${ }^{4}$ Deutsches Konsortium für translationale Krebsforschung (DKTK), Partner SiTe Munich, Munich, Germany.

Received: 18 July 2018 Accepted: 6 November 2018

Published online: 22 November 2018

\section{References}

1. Ajani JA, Winter KA, Gunderson LL, Pedersen J, Benson AB, Thomas CR Jr, et al. Fluorouracil, mitomycin, and radiotherapy vs fluorouracil, cisplatin, and radiotherapy for carcinoma of the anal canal: a randomized controlled trial. JAMA. 2008;299(16):1914-21. https://doi.org/10.1001/jama.299.16.1914.

2. James RD, Glynne-Jones R, Meadows HM, Cunningham D, Myint AS, Saunders MP, et al. Mitomycin or cisplatin chemoradiation with or without maintenance chemotherapy for treatment of squamous-cell carcinoma of the anus (ACT II): a randomised, phase 3, open-label, $2 \times 2$ factorial trial. Lancet Oncol. 2013;14(6):516-24. https://doi.org/10.1016/S14702045(13)70086-X

3. Fuchs WA. Lymphographie und Tumordiagnostik. Berlin; Heidelberg; New York: Springer Verlag; 1965. p. 66.

4. Lee NY, Lu JJ. Target volume delineation and field setup: a practical guide for conformal and intensity modulated radiation therapy. Berlin, Heidelberg: Springer; 2013.

5. Myerson RJ, Garofalo MC, El Naqa I, Abrams RA, Apte A, Bosch WR, et al. Elective clinical target volumes for conformal therapy in anorectal cancer: a radiation therapy oncology group consensus panel contouring atlas. Int J Radiat Oncol Biol Phys. 2009;74(3):824-30. https://doi.org/10.1016/j.jirobp. 2008.08.070.

6. Hirche C, Dresel S, Krempien R, Hünerbein M. Sentinel node biopsy by indocyanine green retention fluorescence detection for inguinal lymph node staging of anal cancer: preliminary experience. Ann Surg Oncol. 2010; 17(9):2357-62. https://doi.org/10.1245/s10434-010-1010-7. 
7. Doniga SG. Die Fluoreszenzfarbstoff-gestützte Sentinel-LymphknotenBiopsie mittels Indocyanin Grün bei solitären Tumoren [@Berlin, Univ.Medizin, Diss. 2013]. Verfügbar unter: http://www.diss.fu-berlin.de/diss/ receive/FUDISS_thesis_000000094271.

8. Dapper H, Habl G, Hirche C, Münch S, Oechsner M, Mayinger M, et al. Dosimetric quantification of the incidental irradiation of the 'true' (deep) ano-inguinal lymphatic drainage of anal cancer patients not described in conventional contouring guidelines. Acta Oncol. 2018:1-6. https://doi.org/ 10.1080/0284186X.2017.1415459.

9. Ortholan C, Resbeut M, Hannoun-Levi J-M, Teissier E, Gerard J-P, Ronchin P, et al. Anal canal cancer: management of inguinal nodes and benefit of prophylactic inguinal irradiation (CORS-03 study). Int J Radiat Oncol Biol Phys. 2012;82(5):1988-95. https://doi.org/10.1016/j.ijrobp.2011.02.010.

10. Blinde SE, Schasfoort R, Mens JW, Verhoef C, Olofsen M, Nuyttens JJ. Inguinal lymph node recurrence in the untreated groin of patients with anal carcinoma. Dis Colon Rectum. 2014;57(5):578-84. https://doi.org/10. 1097/DCR.0000000000000050.

11. Tomaszewski JM, Link E, Leong T, Heriot A, Vazquez M, Chander $S$, et al. Twenty-five-year experience with radical chemoradiation for anal cancer. Int J Radiat Oncol Biol Phys. 2012;83(2):552-8. https://doi.org/10.1016/j.jrobp. 2011.07.007.

12. Nilsson PJ, Svensson C, Goldman S, Ljungqvist O, Glimelius B. Epidermoid anal cancer: a review of a population-based series of 308 consecutive patients treated according to prospective protocols. Int J Radiat Oncol Biol Phys. 2005;61(1):92-102. https://doi.org/10.1016/j.jirobp.2004.03.034.

13. James RD, Pointon RS, Martin S. Local radiotherapy in the management of squamous carcinoma of the anus. Br J Surg. 1985;72(4):282-5.

14. Das P, Bhatia S, Eng C, Ajani JA, Skibber JM, Rodriguez-Bigas MA, et al. Predictors and patterns of recurrence after definitive chemoradiation for anal cancer. Int J Radiat Oncol Biol Phys. 2007;68(3):794-800. https://doi.org/ 10.1016/j.jijobp.2006.12.052.

15. Tomasoa NB, Meulendijks D, Nijkamp J, Cats A, Dewit L. Clinical outcome in patients treated with simultaneous integrated boost - intensity modulated radiation therapy (SIB-IMRT) with and without concurrent chemotherapy for squamous cell carcinoma of the anal canal. Acta Oncol. 2016;55(6):760-6. https://doi.org/10.3109/0284186X.2015.1124141.

16. Bryant AK, Huynh-Le MP, Simpson DR, Murphy JD. Comparative effectiveness of IMRT and 3D comparative effectiveness of IMRT and 3D conformal radiation therapy for anal Cancer. Int J Radiat Oncol Biol Phys. 2017:99.

17. Kachnic LA, Winter K, Myerson RJ, Goodyear MD, Willins J, Esthappan J, et al. RTOG 0529: a phase 2 evaluation of dose-painted intensity modulated radiation therapy in combination with 5 -fluorouracil and mitomycin- $\mathrm{C}$ for the reduction of acute morbidity in carcinoma of the anal canal. Int J Radiat Oncol Biol Phys. 2013;86(1):27-33. https://doi.org/10.1016/j.ijrobp.2012.09.023.

18. Pepek JM, Willett CG, Czito BG. Radiation therapy advances for treatment of anal cancer. J Natl Compr Cancer Netw. 2010;8(1):123-9.

19. Chen AM, Farwell DG, Luu Q, Chen LM, Vijayakumar S, Purdy JA. Marginal misses after postoperative intensity-modulated radiotherapy for head and neck cancer. Int J Radiat Oncol Biol Phys. 2011;80(5):1423-9. https://doi.org/ 10.1016/j.jirobp.2010.04.011.

20. Cannon DM, Lee NY. Recurrence in region of spared parotid gland after definitive intensity-modulated radiotherapy for head and neck cancer. Int J Radiat Oncol Biol Phys. 2008;70(3):660-5. https://doi.org/10.1016/j.jjrobp. 2007.09.018.

21. Marks LB, Yorke ED, Jackson A, ten Haken RK, Constine LS, Eisbruch A, et al. Use of normal tissue complication probability models in the clinic. Int J Radiat Oncol Biol Phys. 2010;76(3 Suppl):S10-9. https://doi.org/10.1016/j. ijrobp.2009.07.1754.

22. Ng M, Leong T, Chander S, Chu J, Kneebone A, Carroll S, et al. Australasian gastrointestinal trials group (AGITG) contouring atlas and planning guidelines for intensity-modulated radiotherapy in anal cancer. Int J Radiat Oncol Biol Phys. 2012;83(5):1455-62. https://doi.org/10.1016/j.jijobp.2011.12.058.

23. R Muirhead, RA Adams, DC Gilbert, M Harrison, R Glynne-Jones, D SebagMontefiore, MA Hawkins. National guidance for IMRT in anal national guidance for IMRT in anal cancer: the CRUKMMRC Oxford Institute for Radiation Oncology, Oxford, UK; School of Medicine, Cardiff University, Cardiff, UK; Sussex Cancer Centre, Royal Sussex County Hospital, Brighton, UK; Mount Vernon Hospital, Northwood, UK; University of Leeds, St James Institute of Oncology, Leeds, UK; 2016 [Stand: 08.08.2018]. Verfügbar unter: http://analimrtguidance. co.uk/national-anal-imrt-guidance-v3.pdf.
24. Aumüller G, Wolff W. Duale Reihe - Anatomie. Duale Reihe 1. Aufl. Stuttgart: Thieme; 2007. Duale Reihe

25. Scher ED, Ahmed I, Yue NJ, Jabbour SK. Technical aspects of radiation therapy for anal cancer. J Gastrointest Oncol. 2014;5(3):198-211. https://doi. org/10.3978/j.issn.2078-6891.2014.026.

26. Mitchell MP, Abboud M, Eng C, Beddar AS, Krishnan S, Delclos ME, et al. Intensity-modulated radiation therapy with concurrent chemotherapy for anal cancer: outcomes and toxicity. Am J Clin Oncol. 2014;37(5):461-6. https://doi.org/10.1097/COC.0b013e31827e52a3.

27. Meulendijks D, Dewit L, Tomasoa NB, van Tinteren H, Beijnen JH, Schellens $J H M$, et al. Chemoradiotherapy with capecitabine for locally advanced anal carcinoma: an alternative treatment option. Br J Cancer. 2014;111(9):172633. https://doi.org/10.1038/bjc.2014.467.

28. Koeck J, Lohr F, Buergy D, Büsing K, Trunk MJ, Wenz F, Mai S. Genital invasion or perigenital spread may pose a risk of marginal misses for Intensity Modulated Radiotherapy (IMRT) in anal cancer: BioMed Central Ltd; 2016.

29. Wright JL, Patil SM, Temple LKF, Minsky BD, Saltz LB, Goodman KA. Squamous cell carcinoma of the anal canal: patterns and predictors of failure and implications for intensity-modulated radiation treatment planning. Int J Radiat Oncol Biol Phys. 2010;78(4):1064-72. https://doi.org/10. 1016/j.jijobp.2009.09.029.

\section{Ready to submit your research? Choose BMC and benefit from:}

- fast, convenient online submission

- thorough peer review by experienced researchers in your field

- rapid publication on acceptance

- support for research data, including large and complex data types

- gold Open Access which fosters wider collaboration and increased citations

- maximum visibility for your research: over $100 \mathrm{M}$ website views per year

At BMC, research is always in progress.

Learn more biomedcentral.com/submissions 